\title{
A novel xeno-free and feeder-cell-free system for human pluripotent stem cell culture
}

\author{
Qihui Wang ${ }^{1,2^{*}}$, Xiaoning Mou ${ }^{1,2^{*}}$, Henghua Cao ${ }^{1}$, Qingzhang Meng ${ }^{1}$, Yanni Ma ${ }^{1}$, Pengcheng Han ${ }^{1,2}$, \\ Junjie Jiang ${ }^{1,2}$, Hao Zhang ${ }^{3}$, Yue Ma ${ }^{1 凶}$ \\ ${ }^{1}$ National Laboratory of Biomacromolecules, Institute of Biophysics, Chinese Academy of Sciences, Beijing 100101, China \\ ${ }^{2}$ Graduate School of the Chinese Academy of Sciences, Beijing 100080, China \\ ${ }^{3}$ Department of Surgery, Cardiovascular Institute, Fu Wai Heart Hospital, Chinese Academy of Medical Sciences and Peking \\ Union Medical College, Beijing 100037, China \\ \ Correspondence: yuema@ibp.ac.cn
}

Received November 2, 2011 Accepted November 29, 2011

\begin{abstract}
While human induced pluripotent stem cells (hiPSCs) have promising applications in regenerative medicine, most of the hiPSC lines available today are not suitable for clinical applications due to contamination with nonhuman materials, such as sialic acid, and potential pathogens from animal-product-containing cell culture systems. Although several xeno-free cell culture systems have been established recently, their use of human fibroblasts as feeders reduces the clinical potential of hiPSCs due to batch-to-batch variation in the feeders and time-consuming preparation processes. In this study, we have developed a xeno-free and feeder-cell-free human embryonic stem cell (hESC)/hiPSC culture system using human plasma and human placenta extracts. The system maintains the self-renewing capacity and pluripotency of hESCs for more than $\mathbf{4 0}$ passages. Human iPSCs were also derived from human dermal fibroblasts using this culture system by overexpressing three transcription factors-Oct4, Sox2 and Nanog. The culture system developed here is inexpensive and suitable for large scale production.
\end{abstract}

KEYWORDS human embryonic stem cells, human induced pluripotent stem cells, reprogramming, xeno-free and feeder-cell-free culture system

\section{INTRODUCTION}

Human somatic cells can be reprogrammed to become pluripotent by forced expression of defined transcription factors (Takahashi et al., 2007; Yu et al., 2007). Human induced pluripotent stem cells (hiPSCs) not only have the capacity to differentiate into the cell lineages of the three germ layers, but also allow for the generation of disease-specific and patient-specific hiPSCs. Many gene-deficient diseasespecific iPSCs have been derived (Park et al., 2008; Lee et al., 2009; Ghodsizadeh et al., 2010; Somers et al., 2010), and disease phenotypes can be greatly ameliorated by transplantation of gene-corrected iPSC-differentiated cells (Hanna et al., 2007; Wernig et al., 2008; Xu et al., 2009), providing proof of concept for future iPSC-based therapies ( $\mathrm{Li}$ and Zhou, 2010; Zhang and Gao, 2010). Patient-specific iPSCs should allow the production of genetically-identical cell populations free of immunological rejection when applied in future cell transplantation therapies. However, some issues have to be resolved before these strategies can be translated into clinical applications. One such issue is the biological safety of these cells. Widely-used culture systems for hiPSCs utilize a medium containing a bovine serum extract, and use either mouse embryonic fibroblasts (MEF) (Takahashi et al., 2007; Yu et al., 2007) as feeders or Matrigel as an extracellular matrix (ECM) (Aasen et al., 2008). It has been reported that human embryonic stem cells (hESCs) can incorporate non-human sialic acid present in the medium and mouse feeder cells or Matrigel matrix (Martin et al., 2005). This contamination could potentially evoke immunorejection in patients and lessen the benefits of autologous cell transplantation therapies. Moreover, hiPSCs could be infected by nonhuman pathogens from the animal products contained in the cell culture system, another risk for human

"These authors contributed equally to the work. 
therapies. Therefore it is necessary to derive and propagate hiPSCs in xeno-free culture conditions. The use of human fibroblasts as feeders is another hurdle for the potential clinical applications of hiPSCs. Feeder cells vary from batch to batch, and this variation diminishes the reproducibility of cell differentiation procedures. In addition, preparation of feeders is time consuming and not suitable for large-scale production.

In the present study, we developed a system for hESCs/ hiPSCs culture composed by xeno-free (XF) medium and feeder-cell-free (FCF) matrix. We used a simple procedure to extract ECM proteins from human placentas. This FCF matrix contains collagen IV, laminin and fibronectin. Next, we developed "XF medium" from human plasma using $\mathrm{NaCl}$ sedimentation. This culture system can support the selfrenewal of hESCs for more than 40 passages. Human iPSCs were also generated from human dermal fibroblasts (HDFs) using this culture system by lentivirus-mediated overexpression of three transcription factors-Oct4, Sox 2 and Nanog ( $\mathrm{Yu}$ et al., 2007). This xeno-free and feeder-cell-free culture system is inexpensive, scalable and easy to produce.

\section{RESULTS AND DISCUSSION}

\section{Establishment of a xeno-free and feeder-cell-free culture system for pluripotent stem cells}

Since the human placenta is a rich and readily-available source of ECM proteins, we modified the urea extraction method used for the preparation of Matrigel (Kibbey, 1994), and developed a simple procedure for extracting ECM proteins from human placentas. After mincing, and sequential washing with distilled water, $\mathrm{NaCl}$ solution and acetic acid, the tissue was digested with pepsin. After extracting with urea, urea was removed by dialysis. The resulting FCF matrix protein solution, like Matrigel, forms a thick gel at $37^{\circ} \mathrm{C}$. Western blotting results showed that pepsin-digested fragments of collagen IV, fibronectin and laminin were present in the FCF matrix (Fig. S1A). Each preparation typically generates about $214 \mathrm{mg}$ matrix proteins from $100 \mathrm{~g}$ placental tissue. We tested the growth of $\mathrm{H} 7$ cells on FCF matrix with MEF-conditioned medium (MEF-CM), and found that about $10 \%$ of the concentration of Matrigel typically used (Xu et al., 2001 ) is sufficient for maintaining the self-renewing capacity of $\mathrm{H} 7 \mathrm{hESCs}$.

To develop a xeno-free culture medium which complies with the biosafety requirements for clinical applications, we first compared three methods for partially purifying human plasma: DEAE chromatography, saturated ammonium sulfate sedimentation and $\mathrm{NaCl}$ sedimentation. These three plasma extracts were evaluated for their ability to support the selfrenewal of undifferentiated $\mathrm{H} 7 \mathrm{hESC}$ s by assessing the nuclear-cytoplasmic ratio of cultured cells, since typical undifferentiated hESCs possess a high nuclear-cytoplasmic ratio (Thomson et al., 1998). Cultured H7 hESCs lost their high nuclear-cytoplasmic ratio after six passages in most of the plasma extracts (data not shown) except those obtained by $\mathrm{NaCl}$ sedimentation. Long term culture demonstrated that hESCs can grow for over 40 passages on FCF matrix in XF medium which contains the $\mathrm{NaCl}$ sedimentation plasma extract and supplements (details in MATERIALS AND METHODS). Four batches of FCF matrix and 11 batches of $\mathrm{XF}$ medium were tested and no obvious variation in the morphology of cultivated hESCs was observed between different batches.

\section{hESCs cultured in XF/FCF system retain their pluripotency}

The widely-used hESC culture system includes Matrigel (MG) as the ECM and MEF-CM (MEF-CM/MG) (Xu et al., 2001). We compared the capacity of the MEF-CM/MG culture system with our novel XF/FCF culture system for maintaining the self-renewing and pluripotent potential of hESCs. In the culture system we developed, $\mathrm{H} 7 \mathrm{hESC}$ s had morphologies resembling those of cells maintained with the MEF-CM/MG culture system (Fig. 1A). After more than 9 months of culture (39 passages), cells maintained in the XF/FCF system had a normal karyotype (Fig. 1B). Fluorescence activated cell sorting (FACS) analysis showed that the percentages of $\mathrm{H} 7$ hESCs expressing Oct4, and surface markers SSEA4, TRA1-60 and TRA-1-81 were not significantly different in the XF/ FCF and MEF-CM/MG culture systems (Fig. 1C). Quantitative reverse transcription-polymerase chain reaction (RT-PCR) analysis confirmed the FACS results (Fig. 1D), indicating that hESCs cultured in the XF/FCF culture system maintained their self-renewing capacity after long term culture.

In order to evaluate the pluripotency of hESCs cultured in the XF/FCF system in vitro, embryoid bodies (EBs) were generated from $\mathrm{H} 7 \mathrm{hESC}$ s cultured in the XF/FCF and MEFCM/MG systems. Results from RT-PCR experiments demonstrated that EBs generated using the two systems all expressed marker genes for the ectoderm (neurofilament heavy chain, NFH), mesoderm (cardiac actin) and endoderm (Thy-1) (Fig. 2A). We also injected undifferentiated hESCs cultured in the XF/FCF culture system into nonobese diabetic/ severe combined immuno-deficient (NOD/SCID) mice to test the differentiation capacity of the cells in vivo. After 10-12 weeks, all injected mice produced teratomas. Teratomas were collected and stained with hematoxylin and eosin (HE). Results showed the presence of tissues originating from each of the three germ layers in the teratomas generated from cells in the XF/FCF system (Fig. 2B). Taken together, these results indicate that our XF/FCF culture system has a similar capacity for supporting self-renewal and pluripotency of hESCs as the MEF-CM/MG culture system. 
A

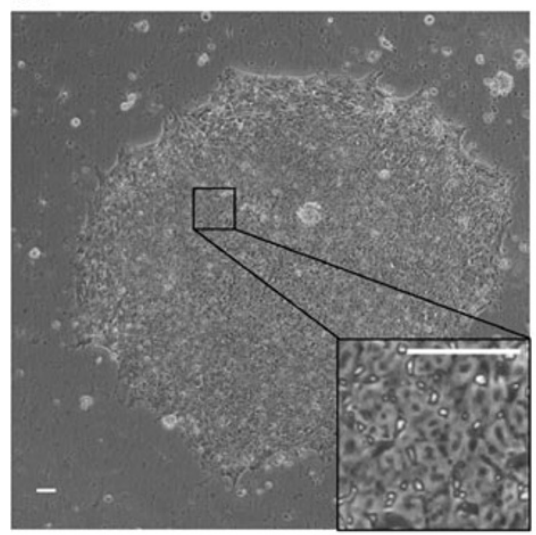

C

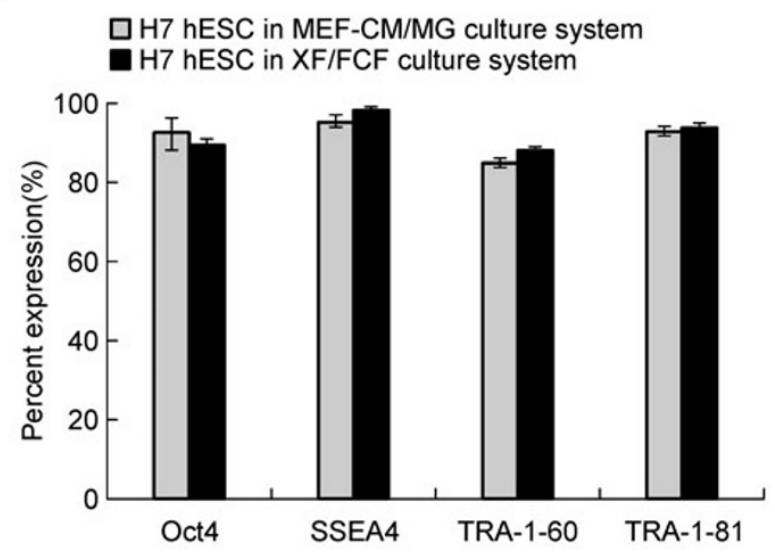

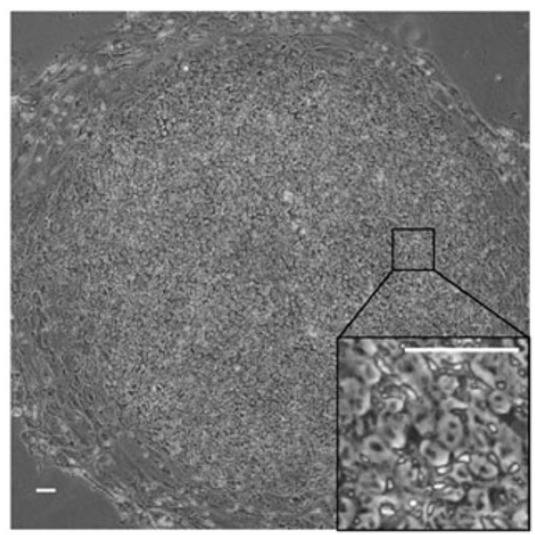

B

\begin{tabular}{|c|c|c|c|c|c|c|}
\hline 11 & 11 & 10 & & & 11 & 18 \\
\hline 1 & 2 & 3 & & & 4 & 5 \\
\hline 81 & 88 & 18 & in & 81 & yt & 18 \\
\hline 6 & 7 & 8 & 9 & 10 & 11 & 12 \\
\hline of & $O A$ & 10 & & 18 & A: & 80 \\
\hline 13 & 14 & 15 & & 16 & 17 & 18 \\
\hline 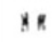 & 88 & & $\leadsto$ & $n A$ & 11 & \\
\hline 19 & 20 & & 21 & 22 & $x$ & $Y$ \\
\hline
\end{tabular}

D

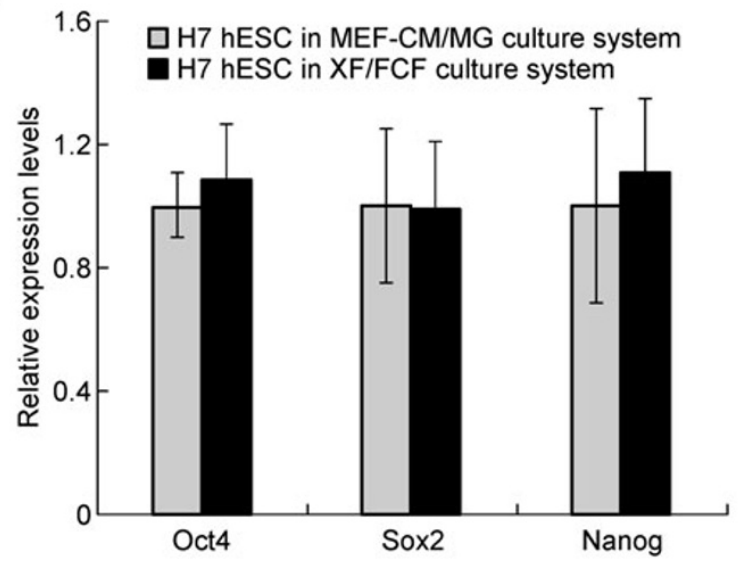

Figure 1. hESCs maintained in the XF/FCF culture system have a normal karyotype and express markers typical of undifferentiated cells. (A) Bright field image of $\mathrm{H} 7 \mathrm{hESC}$ in the MEF-CM/MG culture system (left, passage 48) and XF/FCF culture system (right, $\mathrm{P} 40+8$, the first 40 passages were cultured in the MEF-CM/MG system and the final 8 passages in the XF/FCF system). Inserts show higher magnification of boxed areas. Scale bars, $50 \mu \mathrm{m}$. (B) G-banding analysis of $\mathrm{H} 7 \mathrm{hESC}$ cultured in the XF/FCF culture system for 39 passages. (C) FACS analysis of the expression of Oct4, SSEA4, TRA-1-60 and TRA-1-81 in H7 hESCs in different culture systems. Cells cultured in the XF/FCF system for 28 to 31 passages and in the MEF-CM/MG system for 54 passages were used for analysis. Expression levels were not significantly different between the two systems $(p>0.05, n=3)$. (D) Quantitative RT-PCR analysis of the expression levels of Oct4, Sox2 and Nanog in H7 hESCs maintained in the two culture systems. Cells cultured in the XF/FCF system for 22-29 passages and in the MEF-CM/MG system for 49 passages were used for analysis. The expression levels of the indicated transcripts are given relative to GAPDH expression. Differences in the expression levels of Oct4, Sox2 and Nanog in H7 hESCs maintained in the two culture systems were not significant $(p>0.05, n=3)$.

\section{Reprogramming of human dermal fibroblasts to induced pluripotent stem cells using our XF/FCF culture system}

To generate hiPSCs, HDFs from a 6-year-old boy with ventricular septal defect (obtained with the permission of his guardian) were transduced with lentiviruses bearing Oct4, Sox2 and Nanog genes, and cultured in our XF/FCF culture system. One day after transduction, valproic acid (VPA) was added to the culture (Huangfu et al., 2008). About 10 days after transduction, the morphologies of the cells which survived in the XF/FCF system changed markedly, and both hESC-like and granulate colonies emerged around 20 days post transduction. On day 30 , the efficiency of reprogramming was examined by alkaline phosphatase staining. About seven colonies from $2 \times 10^{5}$ cells were positive for alkaline phosphatase staining. One colony, C1-OSN (Colony No.1 reprogrammed with transcription factors Oct4, Sox2 and Nanog), which had a high nuclear-cytoplasmic ratio, was selected for further analysis (Fig. 3A).

\section{hiPSCs express hESC pluripotent markers and show evidence of nuclear reprogramming}

To characterize the hiPSC line C1-OSN cells generated with our XF/FCF culture system, we examined their expression of undifferentiated hESC markers by immunostaining and 
A

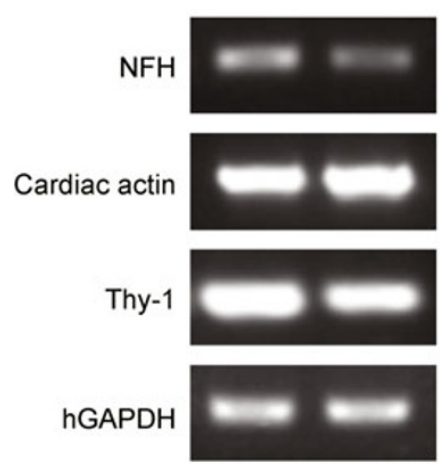

B
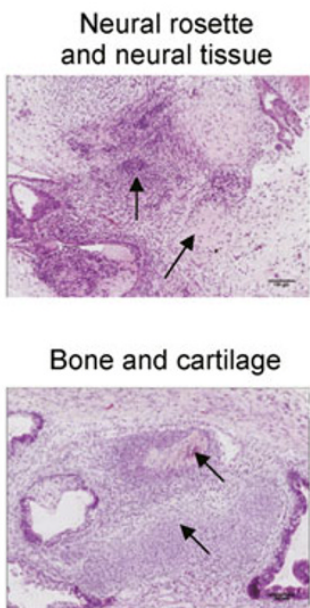

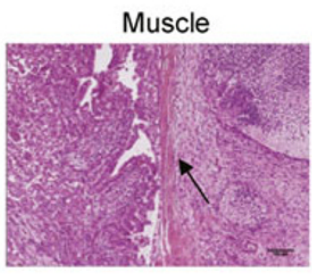

Epithelial luminal structure

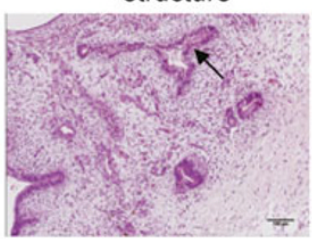

Connective tissue

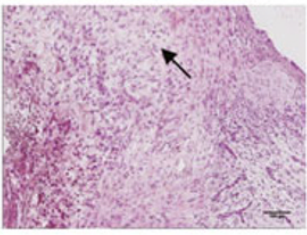

Gut-like

endothelium

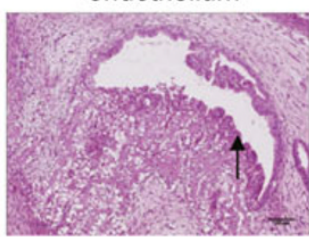

Figure 2. H7 hESCs cultured in the XF/FCF culture system maintain pluripotency in vitro and in vivo. (A) RT-PCR analysis of EBs derived from $\mathrm{H} 7 \mathrm{hESC}$ cultured in the MEF-CM/MG culture system (left) and XF/FCF culture system (right). (B) After 14 passages in the XF/FCF culture system, $\mathrm{H} 7 \mathrm{hESC}$ were injected into NOD/SCID mice. Teratomas were collected and stained with hematoxylin and eosin. Arrows indicate tissues originating from the three embryonic germ layers present in the teratoma. Scale bars, $100 \mu \mathrm{m}$.

A

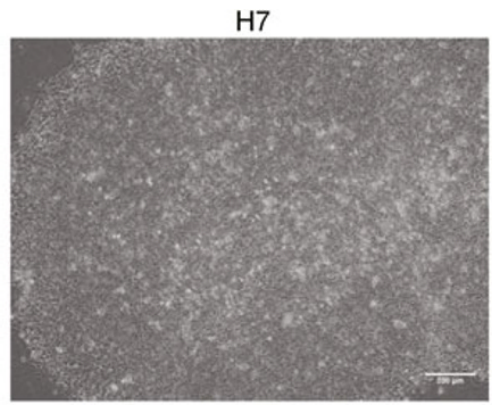

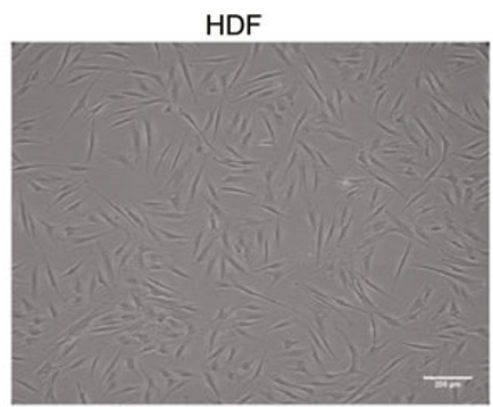

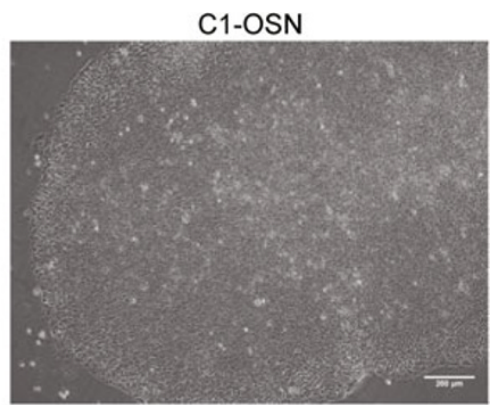

B
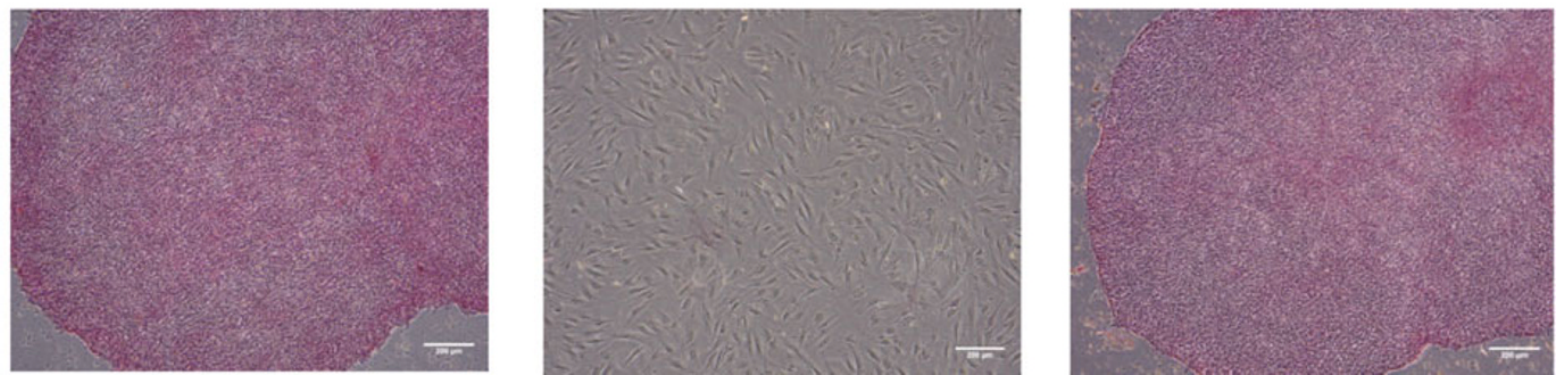

Figure 3. The hiPSCs generated are morphologically similar to hESCs and show alkaline phosphatase expression. (A) Morphology of $\mathrm{H} 7 \mathrm{hESCs}$ (left, passage 37), the parental HDFs (middle) and C1-OSN hiPSCs (right, passage 23). (B) Alkaline phosphatase staining of H7 hESCs (left, passage 37), HDFs (middle) and C1-OSN hiPSCs (right, passage 23). Scale bars, $200 \mu \mathrm{m}$.

quantitative RT-PCR. Results showed that C1-OSN cells were positive for alkaline phosphatase staining (Fig. 3B), and expressed the hESC-specific surface antigens SSEA-4, TRA1-60 and TRA-1-81, and transcription factor Oct4 (Fig. 4A). Quantitative RT-PCR showed that C1-OSN cells expressed undifferentiated hESC-marker genes including Oct4, Sox2 and Nanog at levels equivalent to those of hESCs cultured in the MEF-CM/MG culture system (Fig. 4B).

In order to assess epigenetic reprogramming of C1-OSN, genomic DNA from C1-OSN cells, the parental HDFs and $\mathrm{H} 7$ 
A
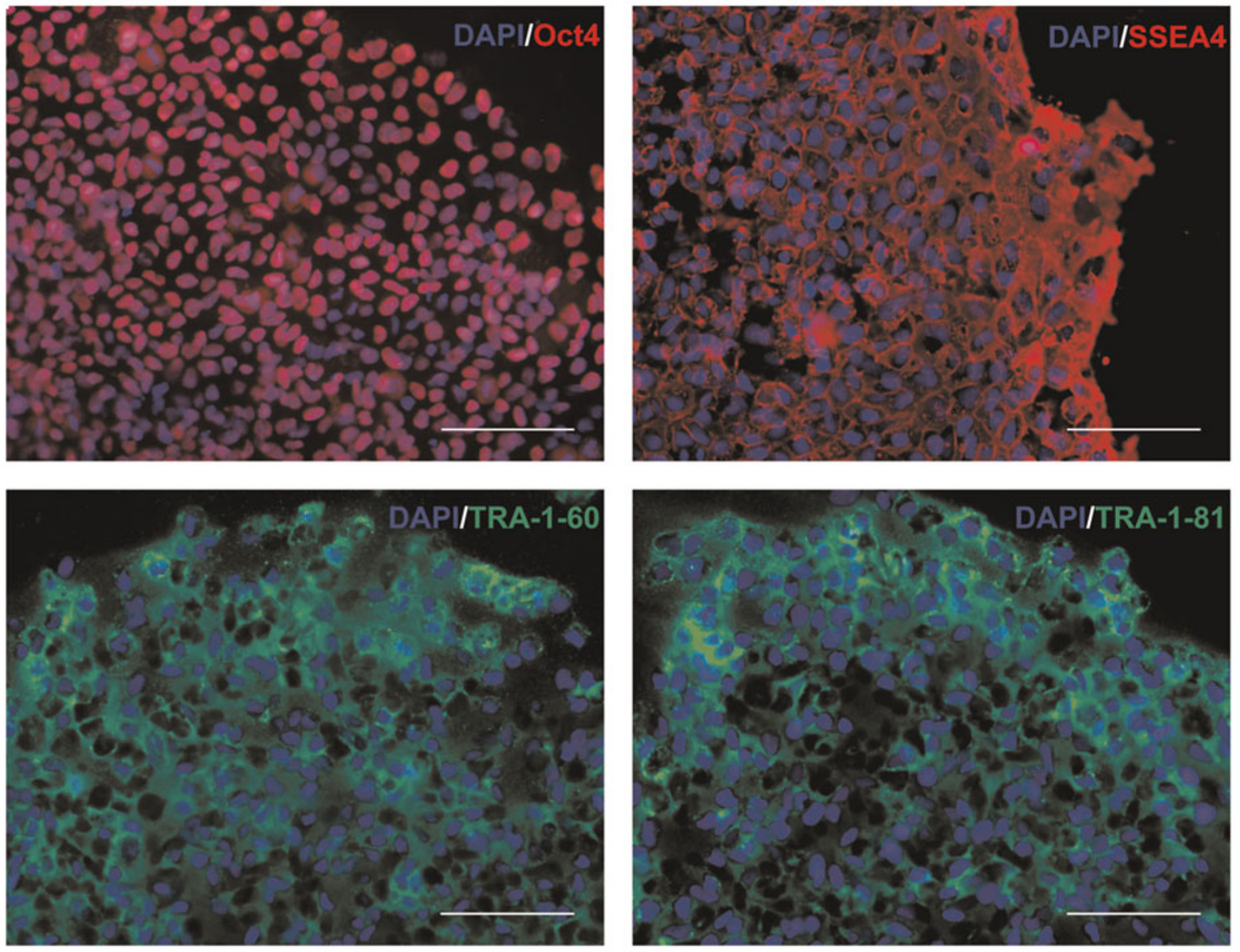

B

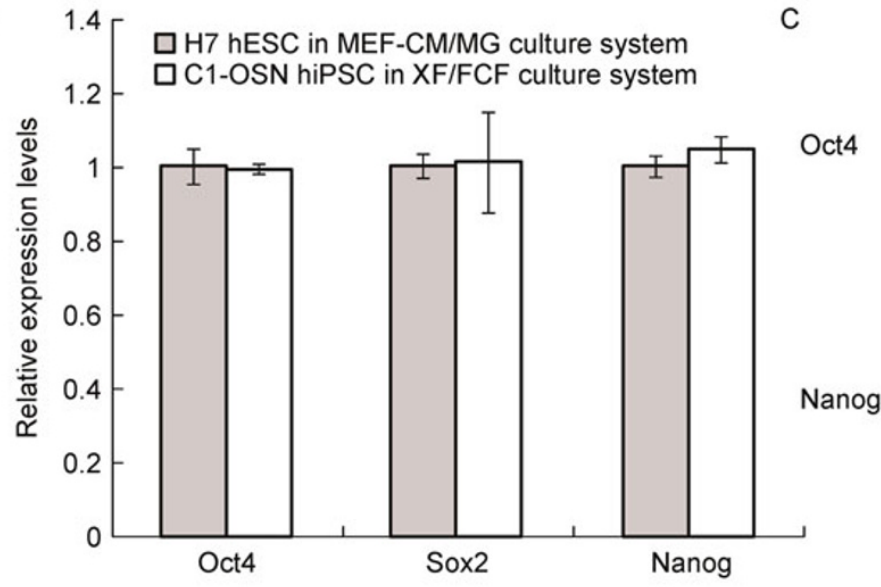

$\mathrm{H} 7$
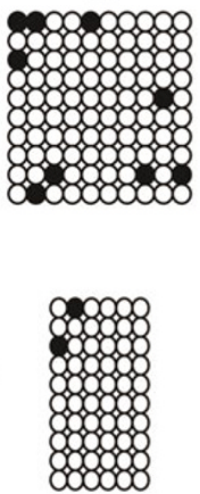

HDF
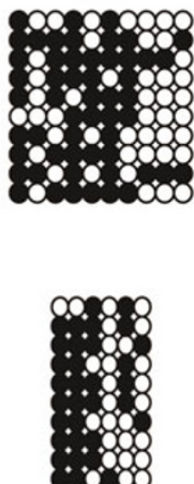

C1-OSN
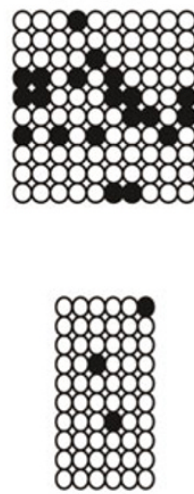

Figure 4. The hiPSCs generated express pluripotent markers and have a methylation status similar to that of hESCs. (A) Immunostaining of C1-OSN cells at passage 11 for the expression of specific markers Oct4, SSEA4, TRA-1-60 and TRA-1-81. Scale bars, $100 \mu \mathrm{m}$. (B) QRT-PCR analysis of the expression levels of pluripotency factors in H7 hESCs (passage 56) and C1-OSN hiPSCs (passage 9). The expression levels of the transcripts indicated are given relative to GAPDH expression. Differences in the expression levels of $\mathrm{H} 7 \mathrm{hESC}$ and C1-OSN hiPSCs were not significant $(p>0.05, n=3)$. (C) Analysis of the methylation status of the Oct4 and Nanog promoters of $\mathrm{H} 7 \mathrm{hESCs}$ (passage 40), HDFs, and C1-OSN hiPSCs (passage 14), by bisulfite sequencing. Open circles indicate unmethylated $\mathrm{CpGs}$, and filled circles indicate methylated CpGs. 
hESCs was analyzed for DNA methylation status. The promoter regions of Oct4 and Nanog were subjected to bisulfite genomic sequencing. Results showed that, as was the case for undifferentiated hESCs, the promoter regions of Oct4 and Nanog in hiPSCs had greater degrees of demethylation than the parental fibroblast lines (Fig. 4C). These results are consistent with the occurrence of epigenetic remodeling during reprogramming by gene transduction (Yamanaka, 2008; Wang and $\mathrm{Na}, 2011$ ). In addition, chromosomal G-banding analysis indicated that C1-OSN hiPSCs had a normal (46, XY) karyotype (Fig. S2).

\section{hiPSCs have multi-lineage differentiation potentials}

The pluripotency of hiPSCs is crucial for their potential application in medicine. We first examined the differentiation ability of C1-OSN cells in vitro. EBs were generated from C1OSN cells, and the expression of markers of the three germ layers and trophoblasts were analyzed by RT-PCR. Results showed that human chorionic gonadotrophin- $\beta$ (hCG- $\beta$ ) (trophoblast lineage), $\alpha$-fetoprotein (AFP) and Thy-1 (endodermal lineage), cardiac actin (mesodermal lineage) and NFH (ectodermal lineage) were all highly expressed (Fig. 5A). The differentiation potential of hiPSCs was also examined in vivo by teratoma formation experiments with NOD/SCID mice. Teratomas were formed in all injected mice about 6 weeks after cell injection. Mice were sacrificed 8-12 weeks after injection. HE staining indicated structures of neural rosettes (ectoderm), skeletal muscles, cartilage, adipose tissues (mesoderm), epithelial lumen and gut-like endothelium (endoderm) (Fig. 5B) were present in the teratomas examined. These results suggest that, like hESCs, hiPSCs generated in the XF/FCF system have multi-lineage differentiation potential.

\section{Verification that C1-OSN hiPSCs are reprogrammed from HDFs}

PCR and short tandem repeat analysis were performed to confirm the origin of the hiPSCs generated in our lab. PCR of genomic DNA from C1-OSN cells showed the integration of all three lentiviruses (Fig. S3). In addition, the patterns of 18 short tandem repeats in C1-OSN hiPSCs and parental HDFs matched completely but were different from those of the hESCs currently cultured in the lab (Table S1). These results indicate that the C1-OSN line generated here is a true hiPSC line, and did not arise by contamination from existing hESC lines in our lab.

hESCs were first derived on MEF by Thomson et al. (Thomson et al., 1998). Later, Xu et al. replaced MEF with Matrigel and MEF-CM (Xu et al., 2001). The major constituents of Matrigel are laminin, collagen, and heparan sulphate proteoglycans. Subsequently, various matrices, including laminin (Beattie et al., 2005), fibronectin (Amit et al., 2004) and vitronectin (Braam et al., 2008), and combinations of laminin, fibronectin, and collagen IV with (Ludwig et al., 2006) or without vitronectin (Draper et al., 2004), were reported to support hESC propagation in different media. Most of these ECM proteins are abundant in the human placenta. We have demonstrated here that ECM protein extracts from human placentas can be readily prepared, and our calculations indicate that more than 60006 -well plates can be coated with matrix proteins extracted from one placenta (of about $500 \mathrm{~g}$ in weight). The human plasma extract made in this study is rich
A

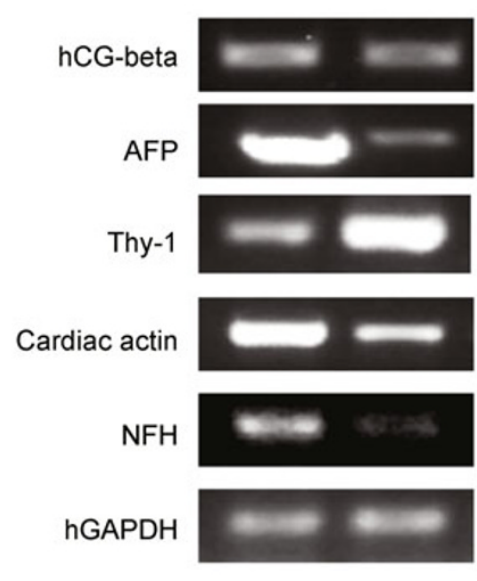

B
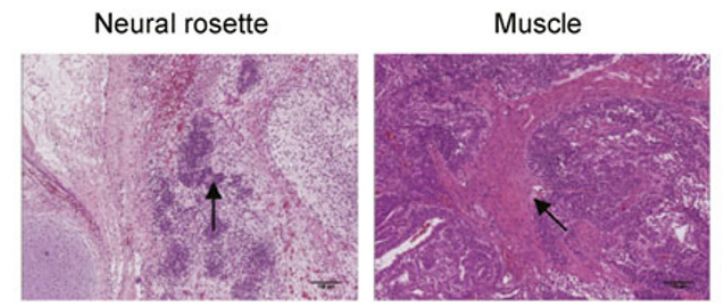

Epithelial luminal structure
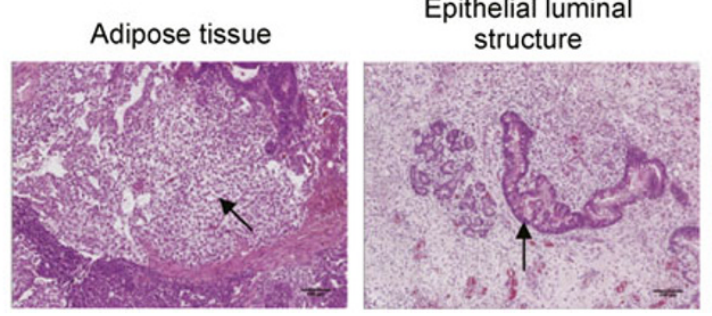

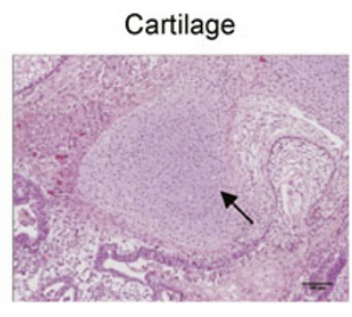

Gut-like endothelium

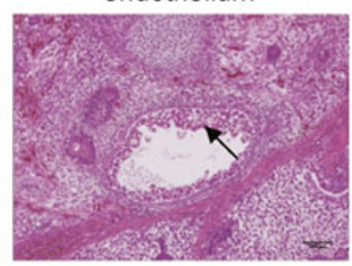

Figure 5. The hiPSCs generated maintain pluripotency in vitro and in vivo. (A) EBs formed by C1-OSN hiPSCs at passage 8 (right) and $\mathrm{H} 7 \mathrm{hESCs}$ at passage 44 (left) express markers for trophoblasts and the three germ layers. (B) After 7 passages in the XF/ FCF culture system, C1-OSN hiPSCs were injected into NOD/SCID mice. Teratomas were collected and stained with hematoxylin and eosin. Arrows indicate tissues originating from the three embryonic germ layers present in the teratoma. Scale bars, $100 \mu \mathrm{m}$. 
in fibronectin and vitronectin, both of which are important ECM proteins. The presence of fibronectin and vitronectin may facilitate the adhesion of hESCs and hiPSCs.

In conclusion, we have established a XF/FCF system for propagating hESCs and hiPSCs. The matrix and medium we developed both originate from human materials, and are devoid of contact with any product originating from animals. The elimination of feeder cells not only removed laborintensive procedures, but also reduced the instability of the culture system. This simple, inexpensive and scalable culture system not only preserves the self-renewal, genetic stability and pluripotency of pluripotent stem cells, but also avoids the biosafety concerns related to the widely used MEF-CM/MG culture systems.

\section{MATERIALS AND METHODS}

\section{Preparation of the matrix and media for hESC/hiPSC culture}

The FCF matrix was derived from human placentas. Human placentas, all of which tested negative for human immunodeficiency virus (HIV), hepatitis B virus (HBV) and syphilis virus (SV), were obtained with the patients' permission. Placentas were separated from the umbilical cord and amnion membrane, minced and then washed sequentially with distilled water, $1 \mathrm{~mol} / \mathrm{L} \mathrm{NaCl}$ solution and $0.5 \mathrm{~mol} / \mathrm{L}$ acetic acid. All procedures were performed at $4^{\circ} \mathrm{C}$. After passing through a sieve, the solid material was digested by pepsin in $0.5 \mathrm{~mol} / \mathrm{L}$ acetic acid and then extracted with a $2 \mathrm{~mol} / \mathrm{L}$ urea solution. Supernatants were collected by centrifuging, and dialyzed against Tris-buffered saline (TBS) containing $0.5 \%$ chloroform, followed by TBS. The resulting solution is the FCF matrix.

The XF medium was derived from human plasma (which tested negative for HIV, HBV and SV; obtained from the blood bank of the 307th General Hospital of PLA). About $4 \mathrm{~L}$ of human plasma (about $1 \mathrm{~L}$ blood of types $\mathrm{A}, \mathrm{B}, \mathrm{O}$ and $\mathrm{AB}$ ) was pooled and $20 \mathrm{mmol} / \mathrm{L} \mathrm{CaCl} \mathrm{Cl}_{2}$ was added. The mixture was incubated at $37^{\circ} \mathrm{C}$ for $2 \mathrm{~h}$, and then stored at $-20^{\circ} \mathrm{C}$ overnight. The thawed mixture was centrifuged, and $\mathrm{NaCl}$ was added to the supernatant to a final concentration of $4.79 \mathrm{~mol} / \mathrm{L}$. The salted-out supernatant was collected and dialyzed against distilled water and DMEM/F12 (Gibco). 1\% nonessential amino acids, $1 \%$ penicillin-streptomycin, $55 \mathrm{nmol} / \mathrm{L} \beta$-mercaptoethanol and supplements (Table S2) were added to the resulting solution to give the XF medium.

\section{Western blotting}

Western blotting was performed with the following antibodies: mouse monoclonal anti-human fibronectin (Sigma), rabbit polyclonal antilaminin (Abcam), rabbit polyclonal anti-collagen IV (Abcam) and rabbit monoclonal anti-human vitronectin (Abcam).

\section{Generation of hiPSCs}

For reprogramming experiments, $2 \times 10^{5} \mathrm{HDFs}$ were transfected with a cocktail of lentiviruses (Ma et al., 2003) which drove the exogenous expression of human Oct4, Sox2 and Nanog genes, and incubated in modified xeno-free medium which lacked TGF $\beta 1$ but contained
$0.5 \mathrm{mmol} / \mathrm{L}$ VPA for 2 weeks. The medium was changed every other day. On day 40, hESC-like cells were selected based on their morphology. To assay the efficiency of reprogramming, infected fibroblasts were fixed and assayed for alkaline phosphatase activity 30 days after infection.

\section{RNA isolation, reverse transcription and polymerase chain reaction}

Total RNA was extracted from cellular samples using TRIzol (Invitrogen) reagent, and cDNA was generated using a SuperScript III First-Strand Synthesis System for RT-PCR kit (Invitrogen). Quantitative RT-PCR was performed using a QuantiFast SYBR Green PCR kit (Qiagen) and detected with a real-time PCR system (Corbett 6200). The statistical significance of differences was analyzed using GraphPad Prism 5. Primer sequences are shown in Table S3.

\section{Alkaline phosphatase staining, immunocytochemistry and fluorescence activated cell sorting}

Alkaline phosphatase staining was performed using an Alkaline Phosphatase Detection kit (Millipore) according to the manufacturer's instructions. For immunocytochemistry, the fixed cells were incubated with Oct4, SSEA4, TRA-1-60, and TRA-1-81 primary antibodies (all from Chemicon). Secondary antibodies used were cyanine 2 (Cy2)conjugated rabbit anti-mouse IgM (Jackson ImmunoResearch) and Rhodamine-conjugated AffiniPure Goat Anti-Mouse IgG (Santa Cruz). Nuclei were stained with DAPI (Sigma). FACS analysis was performed as described (Ludwig et al., 2006).

\section{Short tandem repeat analysis and karyotyping}

Short tandem repeat analysis was performed by the Center of Forensic Sciences, Beijing Genomics Institute. Briefly genomic DNA was used for PCR with AGCU $17+1$ kit (AGCU Scientech Incorporation, Ltd.) and analyzed by ABI PRISM 3100 Genetic analyzer and Gene Mapper v3.5 (Applied Biosystems). Chromosomal G-banding analyses were performed at Peking University Health Science Center. Briefly cells were treated with colcemid (Invitrogen) and stained with Giemsa solution. Spreads were counted for chromosome number and analyzed for banding patterns.

\section{Analysis of DNA methylation}

Genomic DNA was extracted with a DNA Purification Kit (Bioteke, China). Genomic DNA $(2 \mu \mathrm{g})$ was treated with a CpGenome DNA modification kit (Chemicon), according to the manufacturer's instructions. Modified genomic DNA was purified and the promoter regions of human Oct4 and Nanog genes were amplified by nested PCR reactions. Primers used are listed in Table S3. The PCR products were cloned into pGEM T Easy plasmids and transformed into TOP10 cells. Ten clones of each sample were sequenced.

\section{Differentiation of pluripotent stem cells in vitro and in vivo}

Cell colonies were detached by Dispase digestion, then maintained as suspensions in DMEM medium containing $20 \%$ FBS for one week 
to form EBs. The EBs were then seeded on gelatin-coated plates for 7 days. RNA from the formed EBs was purified using TRIzol (Invitrogen) reagent and used to detect the expression of marker genes for the three germ layers. For teratoma formation, cells from one $100 \mathrm{~mm}$ plate $\left(7 \times 10^{6}\right)$ were collected and subcutaneously injected into one NOD/SCID mouse. For each cell line, 4 mice were injected. 8-12 weeks after injection, tissues were collected, sectioned and stained with hematoxylin and eosin.

\section{ACKNOWLEDGEMENTS}

We thank members of the Pathology Lab at the Institute of Biophysics, CAS, for performing H.E. staining. This work was supported by the National High Technology Research and Development Program (863 Program; Grant No. 2006AA02A106), the National Basic Research Program (973 Program; Grant Nos. 2006CB943901, 2010CB945024, and 2011CB965002), the Knowledge Innovation Program of the Chinese Academy of Sciences (KSCX2-YW-R-50), and the National Foundation of Science and Technology (No. 30640005).

Supplementary material is available in the online version of this article at http://dx.doi.org/10.1007/s13238-012-2002-0 and is accessible for authorized users.

\section{ABBREVIATIONS}

EBs, embryoid bodies; ECM, extra-cellular matrix; FACS, fluorescence activated cell sorting; HDFs, human dermal fibroblasts; HE, Hematoxylin and eosin; hESCs, human embryonic stem cells; hiPSCs, human induced pluripotent stem cells; MEF, mouse embryonic fibroblast; MEF-CM, mouse embryonic fibroblastconditioned medium; MEF-CM/MG, mouse embryonic fibroblastconditioned medium/Matrigel; Neu5Gc, N-glycolylneuraminic acid; NOD/SCID, nonobese diabetic/severe combined immuno-deficient; qRT-PCR, quantitative reverse transcription-polymerase chain reaction; TBS, Tris-buffered saline; VPA, valproic acid; XF/FCF culture system, xeno-free and feeder-cell-free culture system

\section{REFERENCES}

Aasen, T., Raya, A., Barrero, M.J., Garreta, E., Consiglio, A., Gonzalez, F., Vassena, R., Bilic, J., Pekarik, V., Tiscornia, G., et al. (2008). Efficient and rapid generation of induced pluripotent stem cells from human keratinocytes. Nat Biotechnol 26, 1276-1284.

Amit, M., Shariki, C., Margulets, V., and Itskovitz-Eldor, J. (2004). Feeder layer- and serum-free culture of human embryonic stem cells. Biol Reprod 70, 837-845.

Beattie, G.M., Lopez, A.D., Bucay, N., Hinton, A., Firpo, M.T., King, C. C., and Hayek, A. (2005). Activin A maintains pluripotency of human embryonic stem cells in the absence of feeder layers. Stem Cells 23, 489-495.

Braam, S.R., Zeinstra, L., Litjens, S., Ward-van Oostwaard, D., van den Brink, S., van Laake, L., Lebrin, F., Kats, P., Hochstenbach, R., Passier, R., et al. (2008). Recombinant vitronectin is a functionally defined substrate that supports human embryonic stem cell selfrenewal via alphavbeta5 integrin. Stem Cells 26, 2257-2265.

Draper, J.S., Moore, H.D., Ruban, L.N., Gokhale, P.J., and Andrews,
P.W. (2004). Culture and characterization of human embryonic stem cells. Stem Cells Dev 13, 325-336.

Ghodsizadeh, A., Taei, A., Totonchi, M., Seifinejad, A., Gourabi, H., Pournasr, B., Aghdami, N., Malekzadeh, R., Almadani, N., Salekdeh, G.H., et al. (2010). Generation of liver disease-specific induced pluripotent stem cells along with efficient differentiation to functional hepatocyte-like cells. Stem Cell Rev 6, 622-632.

Hanna, J., Wernig, M., Markoulaki, S., Sun, C.W., Meissner, A., Cassady, J.P., Beard, C., Brambrink, T., Wu, L.C., Townes, T.M., et al. (2007). Treatment of sickle cell anemia mouse model with iPS cells generated from autologous skin. Science 318, 1920-1923.

Huangfu, D., Osafune, K., Maehr, R., Guo, W., Eijkelenboom, A., Chen, S., Muhlestein, W., and Melton, D.A. (2008). Induction of pluripotent stem cells from primary human fibroblasts with only Oct4 and Sox2. Nat Biotechnol 26, 1269-1275.

Kibbey, M.C. (1994). Maintenance of the EHS sarcoma and Matrigel preparation. Methods Cell Sci 16, 227-230.

Lee, G., Papapetrou, E.P., Kim, H., Chambers, S.M., Tomishima, M. J., Fasano, C.A., Ganat, Y.M., Menon, J., Shimizu, F., Viale, A., et al. (2009). Modelling pathogenesis and treatment of familial dysautonomia using patient-specific iPSCs. Nature 461, 402-406.

Li, Z.K., and Zhou, Q. (2010). Cellular models for disease exploring and drug screening. Protein Cell 1, 355-362.

Ludwig, T.E., Levenstein, M.E., Jones, J.M., Berggren, W.T., Mitchen, E.R., Frane, J.L., Crandall, L.J., Daigh, C.A., Conard, K.R., Piekarczyk, M.S., et al. (2006). Derivation of human embryonic stem cells in defined conditions. Nat Biotechnol 24, 185-187.

Ma, Y., Ramezani, A., Lewis, R., Hawley, R.G., and Thomson, J.A. (2003). High-level sustained transgene expression in human embryonic stem cells using lentiviral vectors. Stem Cells 21, 111-117.

Martin, M.J., Muotri, A., Gage, F., and Varki, A. (2005). Human embryonic stem cells express an immunogenic nonhuman sialic acid. Nat Med 11, 228-232.

Park, I.H., Arora, N., Huo, H., Maherali, N., Ahfeldt, T., Shimamura, A., Lensch, M.W., Cowan, C., Hochedlinger, K., and Daley, G.Q. (2008). Disease-specific induced pluripotent stem cells. Cell 134, 877-886.

Somers, A., Jean, J.C., Sommer, C.A., Omari, A., Ford, C.C., Mills, J. A., Ying, L., Sommer, A.G., Jean, J.M., Smith, B.W., et al. (2010). Generation of transgene-free lung disease-specific human induced pluripotent stem cells using a single excisable lentiviral stem cell cassette. Stem Cells 28, 1728-1740.

Takahashi, K., Tanabe, K., Ohnuki, M., Narita, M., Ichisaka, T., Tomoda, K., and Yamanaka, S. (2007). Induction of pluripotent stem cells from adult human fibroblasts by defined factors. Cell 131, 861-872.

Thomson, J.A., Itskovitz-Eldor, J., Shapiro, S.S., Waknitz, M.A., Swiergiel, J.J., Marshall, V.S., and Jones, J.M. (1998). Embryonic stem cell lines derived from human blastocysts. Science 282 , 1145-1147.

Wang, P., and Na, J. (2011). Mechanism and methods to induce pluripotency. Protein Cell 2, 792-799.

Wernig, M., Zhao, J.P., Pruszak, J., Hedlund, E., Fu, D., Soldner, F., Broccoli, V., Constantine-Paton, M., Isacson, O., and Jaenisch, R. (2008). Neurons derived from reprogrammed fibroblasts functionally integrate into the fetal brain and improve symptoms of rats with Parkinson's disease. Proc Natl Acad Sci USA 105, 5856-5861. 
Xu, C., Inokuma, M.S., Denham, J., Golds, K., Kundu, P., Gold, J.D., and Carpenter, M.K. (2001). Feeder-free growth of undifferentiated human embryonic stem cells. Nat Biotechnol 19, 971-974.

Xu, D., Alipio, Z., Fink, L.M., Adcock, D.M., Yang, J., Ward, D.C., and Ma, Y. (2009). Phenotypic correction of murine hemophilia A using an iPS cell-based therapy. Proc Natl Acad Sci USA 106, 808-813.

Yamanaka, S. (2008). Pluripotency and nuclear reprogramming.
Philos Trans R Soc Lond B Biol Sci 363, 2079-2087.

Yu, J., Vodyanik, M.A., Smuga-Otto, K., Antosiewicz-Bourget, J., Frane, J.L., Tian, S., Nie, J., Jonsdottir, G.A., Ruotti, V., Stewart, R., et al. (2007). Induced pluripotent stem cell lines derived from human somatic cells. Science 318, 1917-1920.

Zhang, X., and Gao, G.F. (2010). Revival of gene therapy. Protein Cell $1,107-108$. 